\title{
CVN: normalización de los currículos científicos
}

\author{
Por José-Manuel Báez, Fernanda Peset, Florencio Núñez y Antonia Ferrer
}

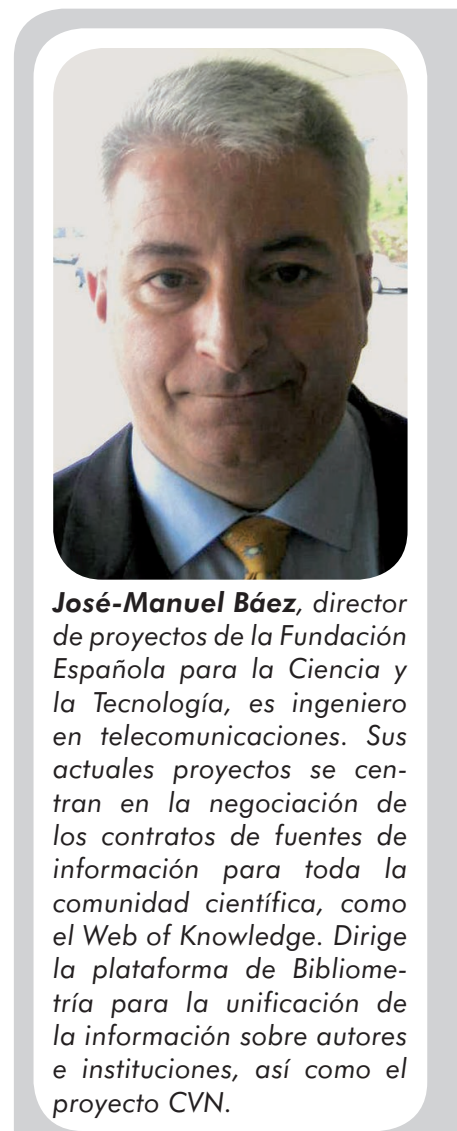

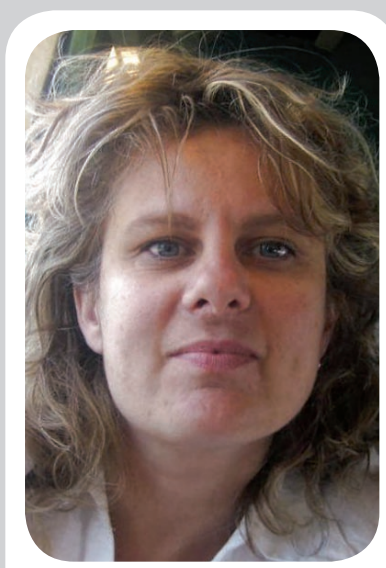

Fernanda Peset, licenciada en geografía e historia y doctora en documentación, es profesora de la Universidad Politécnica de Valencia. Sus líneas de investigación se orientan a la comunicación científica, la normalización en la descripción de la información, y el acceso abierto a la ciencia. Participa en los proyectos IraLIS y E-LIS del del Centro Internacional de Investigación en Estrategia y Prospectiva de la Información (http://www.ciepi.org).

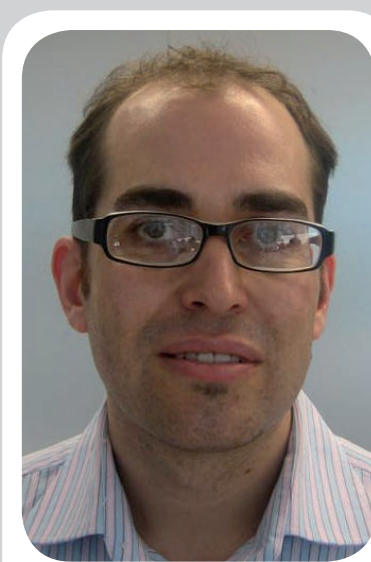

Florencio Núñez, ingeniero técnico en informática por la Universidad Politécnica de Madrid, es técnico de proyectos del departamento de Infraestructuras Tecnológicas de Apoyo a la Investigación, Desarrollo e Innovación $(I+D+I)$ en la Fundación Española para la Ciencia y la Tecnología (Fecyt). Está vinculado al Proyecto de Currículum Vitae Normalizado (CVN) desde el año 2005 (http://cv.normalizado. org/).

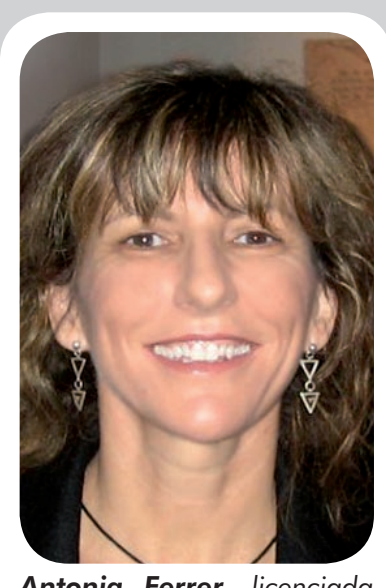

Antonia Ferrer, licenciada en geografía e historia en la especialidad de historia contemporánea por la Universitat de Valencia y doctora en técnicas y métodos actuales en información y documentación, es coordinadora de investigación en Florida Centre de Formació y profesora de la Universidad Politécnica de Valencia. De los últimos proyectos dirigidos cabe destacar el portal de Economía Social EcSocial (http://www. ecsocial.com).

Resumen: Cuanto más estructurado está un sistema científico y mayor es el grado de integración de las entidades que lo forman más eficiente puede hacerse su gestión. Los curricula vitae (CV) de los investigadores son documentos que reúnen datos de personas, instituciones y trabajos, y, vistos en su conjunto, son una privilegiada fuente potencial de información de la actividad científica de un país. Sin embargo si no están almacenados de forma adecuada no es posible explotarlos para realizar estudios sobre ellos. Por otro lado, la inexistencia de un modelo de CV único y normalizado obliga al investigador a rellenar múltiples versiones durante su ejercicio profesional. La Fundación Española para la Ciencia y la Tecnología (Fecyt), junto con las entidades pertenecientes al Sistema español de ciencia, tecnología y empresa (Secte), han puesto en marcha el proyecto Curriculum Vitae Normalizado. Este artículo describe su funcionamiento y destaca los beneficios de su implantación.

Palabras clave: Gestión de la ciencia, Curriculum vitae, Investigadores, Sistema español de ciencia, tecnología y empresa, Secte, Normalización de la información, Sistemas de información curriculares

\section{Title: The standardization of researcher's curricula}

Abstract: The more structured is a scientific system and the greater the degree of integration of its entities the more efficient is its management. Curricula vitae $(\mathrm{CV})$ of researchers are documents that collect data on individuals, institutions and works, and seen as a whole, are a potential source of inside information about the scientific activity of a country. But if they are not stored properly it is not possible to use this knowledge to conduct studies on them. Moreover, the absence of a single standardized $C V$ requires the researchers to fill in multiple versions along their professional practice. The Spanish Foundation for Science and Technology (Fecyt), together with the entities belonging to the Spanish system of science, technology and enterprises (Secte), have initiated the project Standard Curriculum Vitae. This article describes its operation and underlines the benefits of its implementation.

Keywords: Science management, Curriculum vitae, Researchers, Spanish system of science, technology and enterprises, Secte, Information standardization, Curricula information system

Báez, José-Manuel; Peset, Fernanda; Núñez, Florencio; Ferrer, Antonia. “CVN: normalización de los currículos científicos”. En: El profesional de la información, 2008, v. 17, n. 2, marzo-abril, pp. 213-220.

DOI: $10.3145 /$ epi.2008.mar.12 


\section{Introducción}

LA CARRERA DEL CIENTÍFICO, más que otros profesionales, se sustenta sobre su curriculum vitae, un documento con información referente a los múltiples elementos de los que conforman el entramado de la ciencia: datos personales, resultados publicados de la producción científica, patentes, proyectos, grupos de investigación, organizaciones...

Tales elementos están interrelacionados y constituyen redes más o menos estructuradas que en conjunto conforman los sistemas científicos. Podríamos ordenarlos y agruparlos en: personas, instituciones (investigadoras y financiadoras), y resultados, según muestra la figura 1 :

Cada uno de esos componentes tiene a su vez subcomponentes y elementos tanto para difundir como para recibir información. Así:

Personas: directorios, paneles de expertos, listas-e, comunidades virtuales, redes de excelencia, CV.

Instituciones: boletines oficiales, legislación, política científica en otros países, análisis de necesidades, mapas de la ciencia, ciencio-

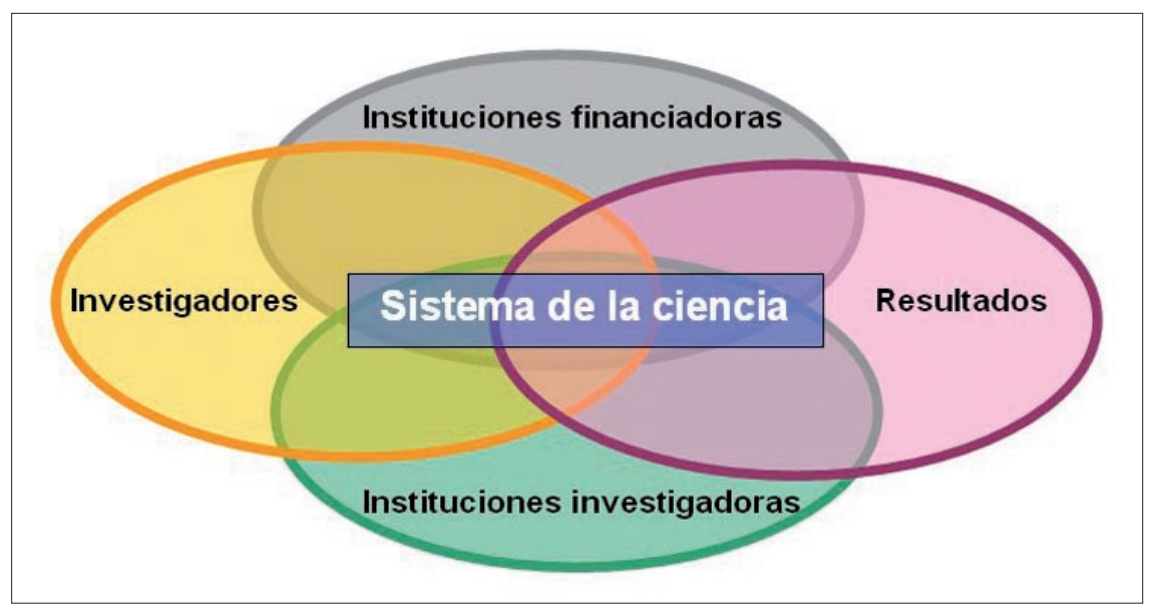

Componentes básicos del sistema de la ciencia

metría, rendimiento de los centros, auditorías, convenios, tranferencia de resultados, OTRIs, patentes, normalización, edición de revistas, organización de congresos, portales, control de calidad.

Resultados: documentos, artículos, bases de datos, repositorios, catálogos, índices...

Los investigadores, en general, mantienen relación con más de un organismo, pues aunque pertenezcan a una institución pueden concurrir a convocatorias de financiación externa (Plan Nacional, autonómicas, privadas...). Para cualquier proceso competitivo se les exige la presentación de una versión actualizada del currículo, el cual viene a ser como un certificado de su capacidad, y deben introducir todos los datos de modo manual en cada uno de los formularios de las instituciones a las que acuden.

Esto supone un importante coste de tiempo pues, a pesar de que la información a introducir cada vez es prácticamente la misma, la extensión media de los currículos de $\mathrm{I}+\mathrm{D}+\mathrm{i}$ es de once páginas para un investigador con cinco años de experiencia.

Puede que un profesor español a lo largo de su carrera tenga

\begin{tabular}{|c|c|}
\hline Curriculum Vitae Europass & Adjunte una fotografía. Suprimir cuando no proceda (ver Instrucciones) \\
\hline \multicolumn{2}{|l|}{ Información personal } \\
\hline $\begin{array}{l}\text { Empleo deseado / familia } \\
\text { profesional }\end{array}$ & Suprimir cuando no proceda (ver instrucciones) \\
\hline \multicolumn{2}{|l|}{ Experiencia laboral } \\
\hline \multicolumn{2}{|l|}{ Educación y formación } \\
\hline \multicolumn{2}{|l|}{$\begin{array}{l}\text { Capacidades y } \\
\text { competencias personales }\end{array}$} \\
\hline Otras informaciones & $\begin{array}{l}\text { Indique en este apartado cualquier otra información que pueda tener rele- } \\
\text { vancia, por ejemplo personas de contacto, referencias, etc. Suprimir cuando } \\
\text { no proceda (ver instrucciones) }\end{array}$ \\
\hline Anexos & $\begin{array}{l}\text { Enumere todos los anexos que haya decidido adjuntar. Suprima cuando no } \\
\text { proceda (ver instrucciones) }\end{array}$ \\
\hline
\end{tabular}

Puede obtenerse más información sobre Europass en http://europass.cedefop.europa.eu () Comunidades Europeas, 2003 - 20060628 
que rellenar los siguientes modelos:

- el de la institución que le haya contratado;

- Ministerio de Educación y Ciencia;

- Agencia Nacional de Evaluación de la Calidad y Acreditación (Aneca);

- Agencia de acreditación para el profesorado contratado;

- Comisión Interministerial de Ciencia y Tecnología (Cicyt)

- organismos de su comunidad autónoma que tengan competencias sobre I+D+i;

- Comisión Nacional Evaluadora de la Actividad Investigadora (Cneai)...

\section{Hacia una mayor estructuración del Secte}

Los organismos del Sistema Español de Ciencia-TecnologíaEmpresa (Secte) han realizado inversiones importantes no sólo para automatizar sus procesos de gestión interna sino para incrementar el acceso y la disponibilidad de la información sobre ciencia y tecnología a través de la Red.

El grado de estructuración e interrelación alcanzado entre los organismos es muy distinto según los países: alto, por ej., en Holanda, y bajo en España, al menos hasta poco tiempo. En nuestro país, además, la existencia de varias administraciones -central, autonómicas- hace más compleja la coordinación.

En este sentido, la labor de la Fundación Española para la Ciencia y la Tecnología (Fecyt) ha sido notable desde su creación en 2001. En sus propias palabras':

"Su misión es prestar un servicio continuado y flexible al sistema español de ciencia-tecnología-sociedad, mediante la identificación de oportunidades y necesidades, y la formulación de propuestas de ac- tuación. Sus objetivos estratégicos son:

- Fomentar actividades de investigación y desarrollo tecnológico de excelencia.

- Propiciar la colaboración entre los agentes del sistema nacional e internacional de ciencia y tecnología.

- Promover la difusión social de la cultura científica, como instrumento de competitividad y mejora de la calidad de vida de los ciudadanos".

Fecyt observó la caótica existencia de múltiples versiones de currículos, pues cada organismo ha desarrollado un sistema ad-hoc para solventar sus necesidades propias, que ha conducido a una situación caracterizada por:

- Dispersión de datos, difíciles de localizar.

- Conjunto no tratable informáticamente.

- Sin homogeneidad de contenidos.

- Datos subjetivamente interpretables.

- Versiones en formatos no tratables (word, pdf).

- Hay que escribir los CVs tantas veces como se piden.

- No se pueden actualizar campos individuales, hay que cambiar todo.

- A veces los datos se introducen $n$ veces según los $n$ miembros de un mismo proyecto.

- Poca fiabilidad de los datos al ser difícil contrastarlos.

- Alto coste de gestión.

- Incompatibilidad con otros modelos internacionales.

Y lo que es peor: muchas veces al final se tiene que ir igualmente con papeles para poder justificar la trayectoria científico-profesional.
Es decir, como consecuencia de que las infraestructuras tecnológicas desarrolladas para cada versión de CV son independientes, no es posible ni explotar la información de modo conjunto ni comunicar los datos entre los sistemas.

En cambio, si se tuvieran en una base de datos estructurada los registros detallados de las actividades científicas de los expertos de un país, se podrían elaborar estadísticas que facilitarían la toma de decisiones y el seguimiento de la financiación pública hasta conocer sus resultados y su impacto social.

\section{EI proyecto Curriculum Vitae Normalizado (CVN)}

Ante la situación descrita, a propuesta del que fue director de la Agencia Nacional de Evaluación y Prospectiva (ANEP), y director general de la Fecyt, Joan X. Comella, en 2005 se inició el proyecto estratégico Curriculum Vitae Normali$z a d o^{3}$. Se trata de establecer un CV estándar que permita al personal investigador y a las instituciones de I+D mantener e intercambiar información científica curricular con todas las ventajas que puede ofrecer la tecnología. La Fecyt es la responsable del proyecto desde el año 2007.

El CVN integra todas las facetas y clases de investigadores en todas las áreas científicas y tecnológicas, incluidas las actividades que se pueden llevar a cabo en bellas artes. Cada individuo debe escoger los campos del CV que necesita en función de su actividad, periodo y destinatario. Su funcionamiento es totalmente electrónico, y los datos se graban en línea. El investigador es quien decide en qué otros sistemas de información curricular (SIC) se replica su currículo para que cualquier actualización sea visible simultáneamente en todos. El formato de exportación es XML.

En este proyecto destaca el estudio previo y las tareas de coordinación llevadas a cabo por la Fecyt 


\section{El CVN responde a los objetivos estratégicos del Sistema Español de Ciencia-Tecnología-Empresa (Secte)}

- Potenciar el papel del sistema público en la generación de conocimiento de carácter fundamental.

- Mejorar la visibilidad y comunicación de los avances de la ciencia y la tecnología en la sociedad española.

- Reforzar la cooperación entre la Administración General del Estado y las Comunidades Autónomas y, en particular, mejorar la coordinación entre el Plan Nacional (PN) y los planes e I+D+i de las CCAA.

- Mejorar la coordinación entre los órganos de gestión del PN, así como perfeccionar los procedimientos de su evaluación y gestión.

- Impulsar la cooperación y coordinación entre las instituciones del sector público de I+D.

- Mejorar la interacción, colaboración y asociación entre el sector público de I+D y el empresarial. con numerosas organizaciones, que muestran la complejidad de nuestro Sistema Español de Ciencia-Tecnología-Empresa (véase el recuadro inferior).

\section{Plan de actuación}

Los siguientes puntos detallan los próximos hitos del proyecto para 2008.

\section{a. Completar los procesos de de- finición de la normativa}

El proyecto CVN se basa en una norma que tienen que cumplir los sistemas informáticos de las entidades participantes. Gracias a este estándar de intercambio CVN-XML los modelos de CV de las entidades del Secte hablarán un mismo lenguaje, es decir, serán interoperables entre sí.

\section{Instituciones consultadas por la Fecyt para el proyecto CVN}

- Comunidades autónomas

- Universidades

- Ministerios: Educación y Ciencia, Industria, Turismo y Comercio, de Sanidad y Consumo, de Trabajo y Asuntos Sociales, de Medio Ambiente, de Fomento, Asuntos Exteriores y Cooperación, de Defensa, de Presidencia, de Economía y Hacienda, de Justicia; en sus Direcciones Generales, Subdirecciones o Secretarías de Estado y los Centros de investigación asociados a ellos como:

- Instituto de Salud Carlos III (Isciii)

- Centro de Investigaciones Energéticas, Medioambientales y Tecnológicas (Ciemat)

- Instituto Geológico y Minero de España (IGME)

- Instituto Español de Oceanografía (IEO)

- Instituto Nacional de Investigación y Tecnología Agraria y Alimentaria (INIA)

- Instituto de Astrofísica de Canarias (IAC)

- Instituto Nacional de Técnica Aeroespacial (INTA)

- Canal de Experiencias Hidrodinámicas de El Pardo (Cehipar)

- Instituto Geográfico Nacional (IGN)

- Centro de Estudios y Experimentación de Obras Públicas (Cedex)

- Centro de Investigaciones Sociológicas (CIS)
- Centro de Estudios Políticos y Constitucionales (Cepco)

- Instituto de Estudios Fiscales (IEF)

- Instituto de Mayores y Servicios Sociales (Imserso)

- Instituto Nacional de Meteorología (INM)

- Instituto Nacional de Toxicología y Ciencias Forenses (Intcf)

- Centro para el Desarrollo Tecnológico Industrial (CDTI)

- Delegación del Gobierno para el Plan Nacional sobre Drogas

- Fundación Cotec para la innovación tecnológica

- Federación Española de Entidades de Innovación y Tecnología (Fedit)

- Asociación de Parques Científicos y Tecnológicos de España (APTE)

- Red de Oficinas de Transferencias de Resultados de la Investigación (OTRI)

- Comisión Nacional Evaluadora de la Actividad Investigadora (Cneai)

- Agencia Nacional de Evaluación de la Calidad y Acreditación (Aneca)

- Conferencia de Rectores de las Universidades Españolas (CRUE) 


\section{a.1. Manual de especificaciones técnicas}

El Manual de especificaciones técnicas es el fruto de 18 meses de trabajo y de 4 comisiones multidisciplinares: 1. Filiación, formación y docencia; 2. Investigación; 3 . Investigación y desarrollo, y Transferencia de tecnología; y 4. Técnica informática.

Por motivos estratégicos durante un año no se ha ampliado a nuevas áreas de conocimiento.

La evolución del Manual requiere tener activos al menos tres de estos grupos de trabajo, analizar los contenidos resultantes de sus reuniones, avanzar en las definiciones y realizar un análisis de los ítems.

El Manual comprende la información que toda clase de investigador, tecnólogo o innovador pueda necesitar para reseñar su trayectoria completa: su actividad y sus resultados. Incluye además la interacción con otras dedicaciones, como, por ejemplo, docente académico o profesional sanitario. Está estructurado en 77 apartados y sub-apartados con unos 1.200 campos en la versión actual.

El modelo contiene los elementos de todas las versiones de $\mathrm{CVs}$ y dependiendo de la manera en que se combinen se obtienen distintos resultados curriculares. Podemos utilizar como símil el alfabeto y la construcción de palabras y frases: el modelo ha sido capaz de fabricar un alfabeto y unas reglas sintácticas y semánticas que permiten la construcción de cualquier palabra o frase.

Durante el año 2008 está previsto que los grupos de trabajo revisen las áreas del Manual de especificaciones técnicas que lo requieran. También se prevé realizar una definición exhaustiva de todos los apartados indicando qué tipos de datos son los indicados para cada uno de ellos.

\section{a.2. Estándar de intercambio CVN-XML}

Es el resultado del análisis de todas las iteraciones que puede tener un apartado curricular del $\mathrm{Ma}$ nual de especificaciones técnicas, abstrayendo sus contenidos.

El estándar de intercambio curricular CVN-XML está basado en un modelo sintético constituido por tres nodos principales:

- Agente,

- Resultados curriculares, y

- Control para la gestión y usos.

Estos tres nodos se modelan con 28 propiedades y sus indicadores asociados. La suma de todos ellos conforma el currículum del investigador en el formato de intercambio CVN-XML.

Durante 2008 la Fecyt tiene previsto ampliar la documentación del modelo sintético, dando cabida a más expresiones curriculares y enriqueciendo la información de los datos existentes.

\section{a.3. Lista maestra de entidades}

Dentro del marco del proyecto CVN, la Fecyt coordina a un grupo de expertos bibliómetras procedentes de varias instituciones españolas: Universidad de Granada, Iedcyt, Parc de Recerca Biomèdica de Barcelona, Universidad Carlos III de Madrid, Universidad de Extremadura y Universidad Politécnica de Valencia, con el objetivo de crear una Lista maestra de entidades - a modo de lista de autoridades normalizadas-, que pondrá a disposición de todo el sistema.

El proceso de creación de esa master list incluye la asociación de un código EAN (European Article Number) a cada una de las entidades que realizan investigación. La Fecyt ha adquirido la licencia para la utilización de un código EAN de 31 dígitos en su versión internacional, no sólo española
$(84352466000110027=$ prefijo + 12 dígitos $=$ entidad +2 dígitos $=$ control). De esta manera cada organismo o empresa contará también con un código de barras que lo describa.

La lista maestra, con tipo, naturaleza, función, organización jerárquica (departamentos y sub-departamentos, si los tiene) y número identificativo, se va a materializar durante 2008.

\section{a.4. Tesauros}

En 2007, la Fecyt, en colaboración con la Agencia Nacional de Evaluación y Prospectiva (ANEP), creó una lista maestra de palabras clave asociadas a las 27 áreas temáticas de la ANEP.

Para el año 2008 está previsto seguir adelante con la labor de definición e incorporación de nuevos términos y clasificaciones en colaboración con la dirección de la Agencia y de los coordinadores de todas sus áreas.

\section{a.5. Tablas de referencia}

Disponer de los datos de los currículos de los investigadores en un formato electrónico estructurado facilitará sin duda las labores de su explotación. Los campos pueden ser de información libre y de información controlada, que es más fácil de explotar. Se está procurando que la estructura de los datos sea compatible con la normativa existente, y es labor de la Fecyt el mantenimiento de los listados contra los que esa información se valida.

Con este fin el proyecto CVN cuenta con más de noventa tablas o listados de dos tipos principales:

- Reconocidos a nivel nacional / internacional, como ejemplo los códigos ISO.

- Creados por la Fecyt al no encontrar un estándar reconocido que pudiera mantener entradas controladas para determinados campos. Durante 2008 se irán incorporando 
nuevos términos, e identificando nuevos campos que puedan estar controlados.

\section{b. Soporte de sistemas}

\section{b.1. Adecuación de sistemas in- formáticos}

El formato de intercambio CVN-XML está basado, como hemos comentado, en una estructura sintética de información. Tiene el beneficio de perdurar en el tiempo, pero también la contrapartida de requerir previamente un periodo de comprensión del modelo seguido para el estándar.

Durante el año 2008 las entidades del sistema español de $\mathrm{I}+\mathrm{D}+\mathrm{i}$ tienen que analizar de forma pormenorizada los datos que contienen sus propios sistemas de información curriculares para adecuarlos y sea posible exportar/importar la información utilizando el formato de intercambio CVN-XML. La Fecyt da soporte a las entidades del Secte, poniendo técnicos especializados para analizar los modelos curriculares de cada una. Se examina el modo de descripción y se identifican las unidades necesarias para la reconstrucción de cualquiera de los ítems existentes que conformarán el CVN-XML. Gracias a la colaboración de la Fecyt, las instituciones serán capaces de adaptar la expresión curricular propia de su sistema al modelo abstracto del estándar de intercambio CVN-XML.

\section{b.2. Certificación de sistemas}

Dado que la esencia del proyecto CVN es la utilización del formato CVN-XML que permite a los investigadores intercambiar información curricular entre instituciones, los sistemas de las entidades del Secte han de entenderse. El formato de intercambio CVN-XML lo crean los sistemas informáticos de las entidades participantes y la $\mathrm{Fe}$ cyt certificará su compatibilidad. Es decir, tiene que certificar que todas las entidades exportan satisfactoriamente datos en formato CVN-XML y auditarlo periódicamente. Para ello está desarrollando la normativa para la acreditación como Entidad Certificada CVN, detallando los pasos a seguir para la conservación de la evaluación positiva, teniendo en cuenta la periodicidad y posibles nuevas versiones del formato de intercambio CVN-XML.

Durante 2007-2008 se realizaron las primeras pruebas de certificación con tres sistemas gestores:

- Sistema de Información Científica de Andalucía (SICA), de la Junta de Andalucía, que integra diez universidades.

- OCU-Universitas XXI investigación, que utilizan trece universidades.

- Sistema de Información Curricular (SIC), del Ministerio de Educación y Ciencia, estrechamente ligado al proyecto CVN, cuyo ámbito es nacional.

La siguiente figura muestra la pantalla de captura de datos desde
Web of Knowledge y la Dirección General de Universidades.

\section{c. Infraestructuras tecnológicas}

Son necesarias para dar soporte al proyecto. Distinguimos dos grandes grupos:

- Básicas: utilizadas por las entidades del sistema para integrarse en el proyecto CVN

- De valor añadido: Aportan funciones adicionales a las Entidades Certificadas CVN

\section{c.1. Infraestructuras básicas}

\section{Web del proyecto}

Es la web utilizada para la divulgación, convirtiéndose en un escaparate en el que se expone el proyecto CVN. La web se encuentra en cinco idiomas.

Consta de dos áreas: una pública dirigida a los investigadores, y otra privada, destinada a las instituciones que se asocian al proyecto.

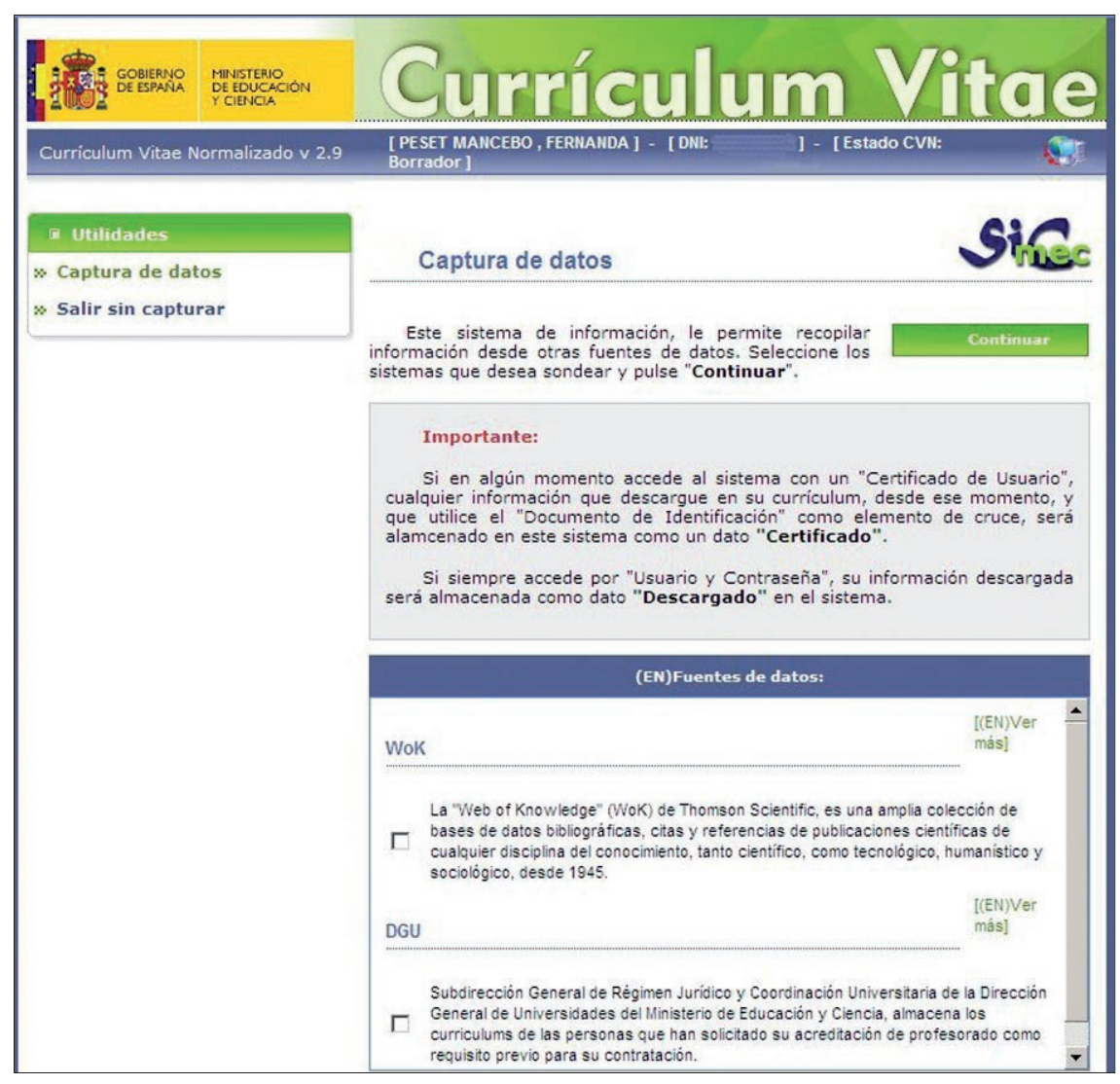

https://segucert.mec.es/sicmecext/entrar.do 


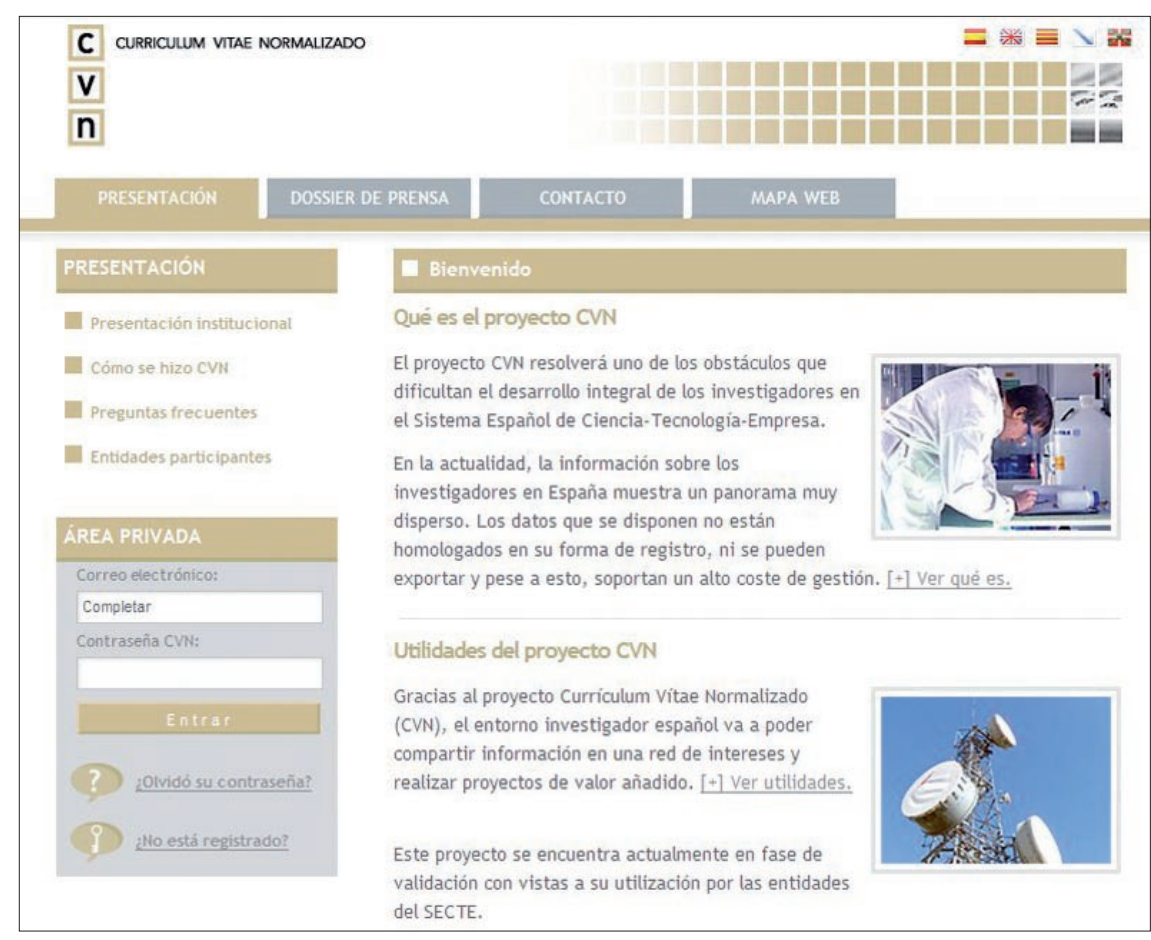

http://cv.normalizado.org/

El área privada es el canal oficial de comunicación entre la Unidad de Soporte del proyecto CVN y las entidades del sistema. Incluye la información de todos los productos necesarios para el correcto entendimiento del proyecto CVN y su estándar de intercambio curricular CVN-XML.

Entorno de pruebas con los sistemas asociados

El proceso de certificación y soporte requiere disponer de una infraestructura que permita intercambiar información sobre las pruebas que se realizan entre las entidades del Secte. Estas entidades tienen distinta naturaleza (administración pública, OPIs, centros tecnológicos, empresas...), por lo que al abrir sus redes informáticas para hacer pruebas se encuentran dificultades de distinta índole. La Fecyt ha creado una plataforma de acceso compartido que permite a todas las entidades conectarse para intercambiar ficheros con otras instituciones.

\section{Validación sintáctica y semántica}

Es un desarrollo web que la Fecyt pondrá a disposición de las entidades participantes y que proporcionará un informe online sobre el estado de adecuación de los curricula vitae. Esta funcionalidad facilita la comprensión de un formato complejo como es el CVN-XML.

Durante 2008 las entidades dispondrán de una herramienta para validar la estructura del XML de CVN, es decir, que se trabaje con objetos válidos en los dos sistemas.

\section{Editor de CVN}

El módulo editor de curriculum vitae normalizado consistirá en una aplicación web gratuita para las entidades participantes que permitirá la importación de un currículo en formato CVN-XML para su edición. También tiene una plantilla para la creación de un nuevo $\mathrm{CV}$ para aquellos usuarios -investigadores de las instituciones- que no dispongan de uno en formato $\mathrm{CVN}$ XML. El software se realizará de forma modular para que aquellas instituciones que lo deseen puedan integrarlo de modo sencillo con su sistema gestor de la investigación.

El editor de CVN requiere un constante mantenimiento para adaptarlo a nuevas versiones curriculares específicas de convocatorias o de especialidades. También debe estar preparado para poder soportar versiones obsoletas del estándar de intercambio CVN-XML, para lo que se prevén desarrollos adicionales de software.

Su puesta en explotación está prevista para el primer semestre de 2008.

\section{c.2. Infraestructuras de valor añadido}

Descarga automática de datos desde bases de datos públicas y licenciadas

Se trata de una aplicación que facilita la carga de parte del currículo importando resultados o experiencias desde bases de datos públicas / licenciadas. Durante 2007 se realizó una descarga de datos desde ISI-Web of Knowledge, dado que la Fecyt gestiona la licencia nacional para el acceso a esta base de datos.

En 2008 se dispondrá de 4 ba-

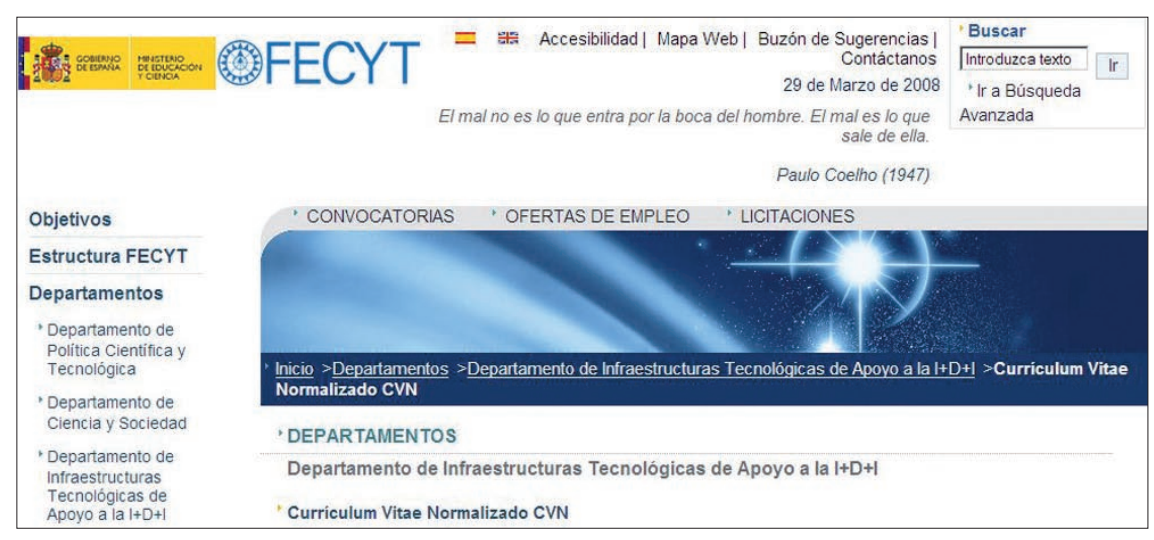


ses de datos más, que se han seleccionado en colaboración con la ANEP.

\section{Módulo de publicación web}

Es un sistema informático que mostrará a través de la web los currículos CVN de los investigadores de las entidades participantes. Permitirá escoger un formato de visualización del currículum de entre los que previamente estén definidos en el portal web del Sistema de Publicación de Currículum.

Un requisito previo a la publicación del CV de un investigador es contar con su autorización.

\section{Gestor de palabras clave/tesauros}

En la actualidad la gestión de palabras clave se hace manualmente. Durante 2008 está previsto desarrollar una aplicación colaborativa accesible desde internet que facilite el proceso de generación de tesauros a los coordinadores de la ANEP. Un supervisor validará las palabras clave y generará las listas maestras para difundir a las entidades.

\section{Conclusiones}

Todavía es muy pronto para valorar el alcance de este proyecto para el sector científico. Como se ha apuntado, la gestión de la ciencia basada en TIC todavía es incipiente en nuestro país, si bien la ley de Administración electrónica y el nuevo Plan Nacional de $I+D+i$ obligan a profundizar en este proceso en un periodo de tiempo corto.

Podemos aventurar que será un hito difícil de superar tanto para los investigadores como individuos, como para las organizaciones que miden la producción de éstos, y las autoridades que diseñan la política científica de nuestro país; pero una vez vencidos los problemas y las inercias, los beneficios serán importantes.

Para los primeros el beneficio reside en contar con datos completos de la actividad constantemente actualizados y que pueden mostrarse en los formatos que cada convocatoria requiera. Las organizaciones se benefician también de este aspecto, pero además de la fiabilidad de los datos y la posibilidad de explotarlos para medir la actividad científica de sus contratados y tomar las decisiones oportunas. Esto mismo, la explotación estadística de los datos, así como su compatibilidad con las normativas existentes, son las ventajas que las autoridades con competencias en $\mathrm{I}+\mathrm{D}+\mathrm{i}$ obtienen del esfuerzo de todo el Secte.

En definitiva, las líneas de futuro que guían este proyecto $\mathrm{CVN}$ a corto plazo serán:

- Dotar al sistema de un formato estándar capaz de adaptar, modelar e integrar todos los diseños curriculares que existen en la actualidad.
- Crear servicios de valor añadido que permitan a las entidades del sistema mejorar sus modelos de gestión de la $\mathrm{I}+\mathrm{D}+\mathrm{i}$

- Utilizar el CVN en las convocatorias del Plan Nacional de $I+D+i$ y en el proyecto de Ventanilla Única de $I+D+i$.

\section{Notas}

1. Definición y objetivos estratégicos. Consulta: 5 de febrero de 2008.

http://www.fecyt.es/fecyt/seleccionarMenu1. do? strRutaNivell $=;$ Objetivos \& tc =objetivos_es trategicos

2. Europass. Consulta: 10 de febrero de 2008. http://europass.cedefop.europa.eu/europass/preview.action?locale_id $=11$

3. CVN Curriculum Vitae Normalizado. Consulta: 8 de enero de 2008 .

http://cv.normalizado.org/

\section{Bibliografía}

Baiget, Tomàs; Rodríguez-Gairín, JosepManuel; Peset, Fernanda; Subirats, Imma; Ferrer, Antonia. "Normalización de la información: la aportación de IraLIS". En: El profesional de la información, 2007, noviembre-diciembre, v. 16, n. 6 , pp. 636-643.

Codina, Lluís. "Motores de búsqueda para usos académicos: ¿cambio de paradigma?”. En: Anиаrio ThinkEPI, n. 1, 2006.

http://www.thinkepi.net/repositorio/motores-debusqueda-para-usos-academicos-¿cambio-deparadigmal

Ontalba-Ruipérez, José-Antonio. "Normalización de campos en bibliometría. Acciones de la Fecyt". En: El profesional de la información, 2007, julio-agosto, v. 16, n. 4, pp. 381-383.

Peset, Fernanda. "La evaluación de los científicos en el currículo para la acreditación nacional". En: Anuario ThinkEPI, 2008.

http://www.thinkepi.net/repositorio/la-evaluacion-de-los-cientificos-en-el-curriculo-para-laacreditacion-nacional/

\section{Suscripciones}

Renovar (o comenzar) la suscripción a "El profesional de la información" es ágil y sencillo.

Usted puede gestionar online su suscripción conectándose a esta web: http://www.elprofesionaldelainformacion.com/suscripciones.html

Si lo desea puede comunicar con nosotros dirigiéndose a: suscripciones@elprofesionaldelainformacion.com o al teléfono: +34-609352954 